\title{
In vivo estimation of water diffusivity in occluded human skin using terahertz reflection spectroscopy
}

Qiushuo Sun1 | Rayko I. Stantchev1 | Jiarui Wang1 | Edward P.J. Parrott1 | Alan Cottenden2 | Tor-Wo Chiu3 | Anil T. Ahuja4 | Emma Pickwell- MacPherson*,1,5

Department of Electronic Engineering, Chinese University of Hong Kong, Hong Kong, China

2 Department of Medical Physics and Biomedical Engineering, University College London, London, UK

3 Division of Plastic Reconstructive and Aesthetic Surgery, Department of Surgery, Chinese University of Hong Kong, Hong Kong, China

Department of Imaging and Interventional Radiology, Chinese University of Hong Kong, Hong Kong, China

5 Department of Physics, University of Warwick, Coventry, UK

\section{Correspondence}

Emma Pickwell-MacPherson, Department of Physics, University of Warwick, Gibbet Hill Road, CV4 7AL, Coventry, UK

Email: E.MacPherson@warwick.ac.uk

\section{(1)
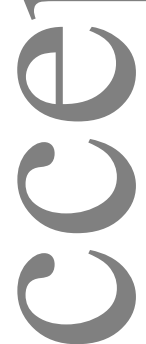 \\ 1 INTRODUCTION}

\subsection{Importance of skin occlusion tests}

The stratum corneum (SC) is the outermost layer of human skin and determines the water barrier function [1]. When skin is occluded, water from inside the SC cannot diffuse to the outside; consequently the water concentration inside increases and the SC starts to swell [2,3]. Skin occlusion tests help to study the permeability, penetration and barrier functions of human skin and could be a powerful tool to monitor skin health. For example, W. Pan et al have used capacitive

KEYWORDS
Water diffusion and the concentration profile within the skin significantly affect the surrounding chemical absorption and molecular synthesis. Occluding the skin causes water to accumulate in the top layer of the skin (the stratum corneum) and also affects the water diffusivity. Scar treatments such as silicone gel and silicone sheets make use of occlusion to increase skin hydration. However with existing techniques, it is not possible to quantitatively measure the diffusivity of the water during occlusion: current methods determine water diffusivity by measuring the water evaporated through the skin and thus require the skin to breathe. In this work we use the high sensitivity of terahertz light to water to study how the water content in the stratum corneum changes upon occlusion. From our measurements, we can solve the diffusion equations in the stratum corneum to deduce the water concentration profile in occluded skin and subsequently to determine the diffusivity. To our knowledge this is the first work showing how the diffusivity of human skin can be measured during occlusion and we envisage this paper as being used as a guide for non-invasively determining the diffusivity of occluded human skin in vivo.

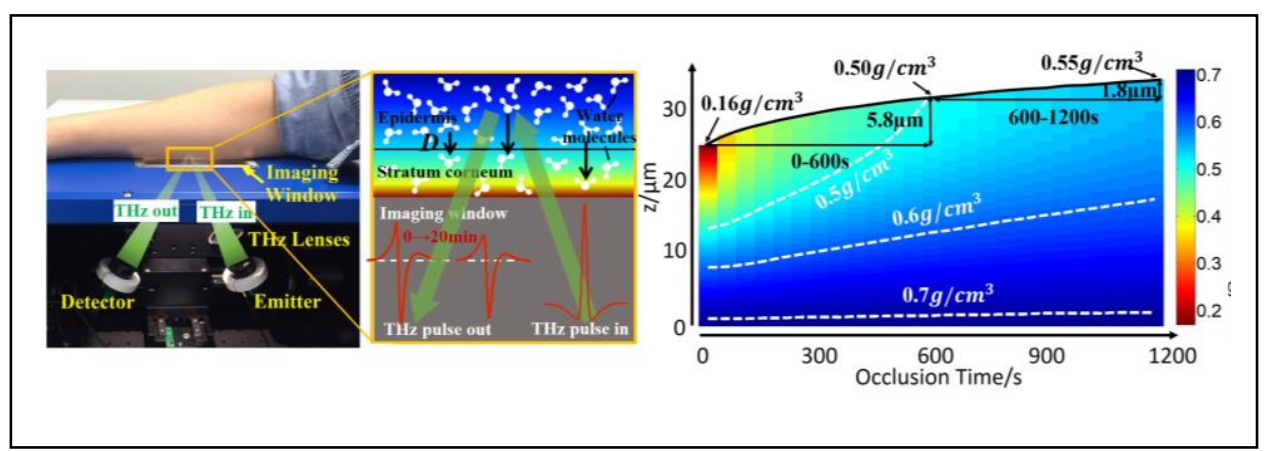

Water diffusivity, skin occlusion, in vivo imaging, terahertz reflection spectroscopy

contact imaging to assess skin damage by comparing the occlusion curves before and after intensive washes, tape stripping and sodium lauryl sulphate irritation [4]. Indeed, skin occlusion is particularly helpful for improving the healing processes of hypertrophic scars and keloids [5]; it is thought that the occlusion and subsequent hydration caused by the occlusive dressing are the main reasons for the improved healing speed [6]. Additionally, occlusion affects molecules in the skin: it enhances the absorption of most chemicals and affects the amounts of RNA, DNA and collagen being synthesized [7].

\section{2 | Fick's law}

This article has been accepted for publication and undergone full peer review but has not been through the copyediting, typesetting, pagination and proofreading process, which may lead to differences between this version and the Version of Record. Please cite this article as doi: $10.1002 /$ jbio. 201800145 
Water motion in skin can be described by Fick's law [4,8-12], in which the water concentration is defined as a function of time and depth in the skin. For hydrated skin, it is important o note that the thickness of the SC changes with the hydration level in it [1]. The thickness of the SC on the volar forearm at steady state is in the range of 15-30 $\mu \mathrm{m}$ [12-16]. When skin is hydrated, the SC swells outwards perpendicularly to the surface of the skin and this phenomenon has been confirmed by in vivo measurements using confocal Raman spectroscopy $[13,16]$. It has been observed that the thickness of corneocytes cells increases from $<0.5 \mu \mathrm{m}$ to $\sim 3 \mu \mathrm{m}$ with the hydration level changing from $20 \%$ to $300 \%$ [17]. This significant change of SC thickness needs to be considered in order to accurately predict the motion of water inside the skin. A dynamic SC swelling model was proposed by $\mathrm{Li}$ et al to simulate water diffusive activity and dynamic thickness change of the SC [11] Existing approaches to measure the hydration of skin such as using a Corneometer can be inaccurate if extra ions (such as sodium chloride) are present as the measurement also depends on the conductivity. Another drawback is that the penetration depth of the measurement is only up to $40 \mu \mathrm{m}$ [18].

\section{3 | Water diffusivity}

Water diffusivity $(D)$ is an essential parameter in Fick's law, it describes how much water passes through a membrane per unit time. By knowing the water diffusivity, the dynamic water flux and concentration profile can be determined. Most of the in vivo methods to determine diffusivity of skin use evaporimeters to measure the transepidermal water loss (TEWL), thereby allowing the substitution of the TEWL value into Fick's law in order to calculate $D[9,10,12,19,20]$. Optical coherence tomography (OCT) can also be used to evaluate the water diffusion velocity in the epidermal and dermal layers [21], but this does not give information about the SC and water barrier function.

To our knowledge, so far there have been no studies investigating how $D$ changes upon occlusion. This is essential to study the transepidermal diffusion of water and molecules in skin and for better development of skin treatments. Traditional TEWL methods become invalid under occlusion as skin cannot breathe. Therefore, in this work we develop a method to measure water diffusivity in occluded human skin real time using terahertz reflection spectroscopy.

\section{4 | Terahertz time domain reflection spectroscopy}

Terahertz $(\mathrm{THz})$ light $\left(1 \mathrm{THz}=10^{12} \mathrm{~Hz}\right)$ is located between the microwave and infrared region of the electromagnetic spectrum with a frequency range from 0.1 to $10 \mathrm{THz}$. THz technology has shown potential in various biological applications due to its non-ionizing feature and $\mathrm{THz}$ time domain reflection spectroscopy (THz-TDRS) is able to discriminate between different types of tissue, including skin, muscle and fat samples as well as discriminate between normal and cancerous tissues, the contrast mechanism is thought to be primarily from the water concentration variation
[22]. $\mathrm{THz}$ light is strongly absorbed by hydrogen bonds in water [22], making it extremely sensitive to water content, and consequently able to noninvasively monitor subtle hydration changes in human skin in vivo. Importantly, in vivo THz-TDRS can also realize real-time measurements. In our recent work, we have shown that skin occlusion leads to a significant time-dependent reduction in the amplitude of the $\mathrm{THz}$ signal [23]: the next step is to make this into a useful diagnostic tool. Bennett et al [24] proposed a stratified media model to simulate the interaction between $\mathrm{THz}$ waves and bio-tissue that is well suited for THz-TDRS studies as the subtle change in the hydration level of each skin layer can be obtained from the reflectivity information, thereby giving more insight about the water concentration profile at any depth in the skin rather than only the integrated $\mathrm{THz}$ reflectivity. There is great potential therefore, to use $\mathrm{THz}$ imaging to measure skin hydration profiles. Furthermore, $\mathrm{THz}$ imaging is not adversely affected by variation in conductivity and is also able to penetrate into the skin deeper than existing approaches such as the corneometer. In general, the penetration depth of $\mathrm{THz}$ light in water is around 40$160 \mu \mathrm{m}$ depending on the frequency [25]. For example, adipose tissue with a high fat content will have a deeper penetration depth since it will have a lower water content. $\mathrm{THz}$ reflections used to determine the thickness of the SC on the palm of 20 volunteers were calculated to be on average $170 \mu \mathrm{m}$ deep in a study by Pickwell et al[26] suggesting $\mathrm{THz}$ light is able to penetrate at least this far in skin on the palm.

\section{5 | This work}

Our recently published groundwork [23] identifies the sensitivity of $\mathrm{THz}$ light to skin occlusion and gives a fitting and modelling method to compensate for occlusion effects when imaging over an area of skin. In this paper, we significantly build on this previous work and show that the $\mathrm{THz}$ response during skin occlusion can be used to determine the water diffusivity and dynamic water concentration distribution during skin occlusion in vivo. We explain how the diffusivity can be extracted from $\mathrm{THz}$ data by employing stratified media theory [24] and a SC swelling model [11] to acquire the water concentration profile in SC as a function of depth and occlusion time. The absolute values of the diffusivity and the water concentration are estimated instead of relative values. We show that in vivo THz-TDRS is a sensitive and quantitative tool able to capture the subtle hydration level changes in skin induced by occlusion effects and has the potential to be useful for developing skin treatments and monitoring wound healing. This is the first work showing how it is even possible to measure diffusivity in the occluded state as other existing techniques rely on the TEWL. Our proposed method provides new information that will be useful for others conducting further skin studies.

\section{2 | EXPERIMENTAL}

\section{1 | Stratum corneum model and finite difference method}


Eqn (1) describes the dynamic water transport in the SC, it has the same form as the convection and diffusion equation [11]

$D(t) \frac{\partial^{2} W(z, t)}{\partial z^{2}}-u(t) \frac{\partial W(z, t)}{\partial z}=\frac{\partial W(z, t)}{\partial t}$

$W(z, t)\left(\mathrm{g} / \mathrm{cm}^{3}\right)$ is the water concentration as a function of depth in skin $z(\mathrm{~cm})$ (with $z=0$ being defined as the boundary of the SC with the epidermis) and time $t(s) . u(t)(\mathrm{cm} / \mathrm{s})$ is the water convection velocity at time $t$, it determines the speed of SC swelling. $D(t)\left(\mathrm{cm}^{2} / \mathrm{s}\right)$ is the water diffusivity, a detailed definition of $D$ is given in the next section.

When skin is in a steady state, the water distribution in skin doesn't change with time, hence the water concentration $W(z, t)$ is only a function of depth $z$. By solving Eqn (1) we acquire the initial water distribution in $\mathrm{SC}$ :

$$
W(z, 0)=1+\left(W_{B 0}-1\right)\left(\frac{W_{S 0}-1}{W_{B 0}-1}\right)^{\frac{z}{L_{0}}}
$$

\section{(2)}

Where $W_{s 0}\left(\mathrm{~g} / \mathrm{cm}^{3}\right)$ and $W_{B 0}\left(\mathrm{~g} / \mathrm{cm}^{3}\right)$ are the initial water concentration at surface and bottom interfaces of SC respectively. $L_{0}(\mathrm{~cm})$ is the initial thickness of SC. When skin is under occlusion, assuming the water concentration at the deeper interface of SC keeps unchanged and the water flux on skin surface decreases to zero $[4,7,13,16,27]$, then the two boundary conditions of occluded SC are defined as:

$$
\left\{\begin{array}{l}
W(0, t)=W_{B 0} \\
-\left.D(t) \frac{\partial W(z, t)}{\partial z}\right|_{z=L(t)}=0
\end{array}\right.
$$

$\mathrm{L}(t)$ is the dynamic thickness of SC. The swelling velocity of the SC is calculated via Eqn (4), which is derived based on the equations in [11]; it equals the convection velocity $u$ :

$$
u(t)=\frac{d L(t)}{d t}=\left.\frac{D(t)}{W_{B 0}-1} \frac{\partial W(z, t)}{\partial z}\right|_{z=0}
$$

With knowledge of the initial parameters $\left(L_{0}, W_{s 0}, W_{B 0}\right)$ of skin and function of $D$, and by solving Eqn (1-4) the water concentration $W(z, t)$ in the SC is calculated. Here, rather than using the commercial software mentioned in ref [11], we use a finite difference method [28] to calculate the numerical solution of Equations (1-4). The computational process is shown in the flow chart in Figure S1. A linear increase and a constant water distribution is assumed in the epidermis and dermis, respectively, as shown in Figure S2.

\subsection{Definition of water diffusivity}

When skin is occluded, the water diffusivity is needed to calculate the dynamic water concentration profile and subsequently the $\mathrm{THz}$ reflectivity. Consequently, the functions of $D$ have to be assumed in the first place. As the water gradient in the SC drives the water diffusion from inside the skin to the outside environment [1], we define three functions for $D$ : a constant, positively correlated with the water concentration at the skin surface and positively correlated with the water concentration difference between the two interfaces of SC. Similarly to in references [19,29], exponential correlations are also applied here. We substitute each one of the functions into the computational loop shown in Figure S1. By comparing the fitting results from these three functions, the best function that describes the change on $D$ was determined.

$$
\begin{aligned}
& F_{1}=D_{0} \\
& F_{2}(W(t))=D_{1} e^{\alpha_{1} W(z=L(t), t)}, \quad D_{1}, \alpha_{1} \geq 0 \\
& F_{3}(\Delta W(t))=D_{2} e^{\alpha_{2}[W(z=0, t)-W(z=L(t), t)]}, \quad D_{2}, \alpha_{2} \geq 0
\end{aligned}
$$

\subsection{THz-TDRS in vivo measurements and reflectivity calculation}

The $\mathrm{THz}$ in vivo measurements were performed at the Chinese University of Hong Kong (CUHK) using a time domain $\mathrm{THz}$ reflection system (Menlo Systems $\mathrm{GmbH}$, Planegg, Germany). The study was approved by the Joint CUHK-NTEC Clinical Research Ethics Committee and written informed consent was obtained from all volunteers to partake in the study. In our setup, the $\mathrm{THz}$ waves are incident on a quartz imaging window with the incident angle $\theta_{\text {air }}=30^{\circ}$ (the beam size is around $8 \mathrm{~mm}$ ) and the area of interest is placed onto the window, illustrated in Figure 1(a). Before measurement, subjects sat in the lab for 20 minutes to acclimatize to the room temperature and humidity. Then they placed their volar forearm on the imaging window for 20 minutes and remained still whilst optics were raster scanned to record the reflected $\mathrm{THz}$ signal from one point of the volar forearm with a speed of $4 \mathrm{~Hz}$, the first measurement was taken immediately after the skin made contact with the imaging window. Five healthy subjects (three males and two females) were measured, with ages in the range of 25-37. The detailed data processing method is explained in our previous work [30]. The reflectivity $R_{\text {skin }}$ of the window-skin interface is calculated by Eqn (6) (for s-polarized light) [24]:

$$
R_{\text {meas }}=\left|\frac{E_{\text {skin }}(\omega)}{E_{\text {air }}(\omega)} r_{\text {quartz-air }}\right|^{2}=\left|\frac{Z_{\text {skin }}-\frac{1}{\cos \theta_{\text {quartz }}} \sqrt{\frac{\mu_{0}}{\varepsilon_{0} \varepsilon_{\text {quartz }}}}}{Z_{\text {skin }}+\frac{1}{\cos \theta_{\text {quartz }}} \sqrt{\frac{\mu_{0}}{\varepsilon_{0} \varepsilon_{\text {quartz }}}}}\right|^{2}=R_{\text {cal }}
$$

On the left-hand side, $E_{\text {skin }}(\omega)$ and $E_{\text {air }}(\omega)$ are the received $\mathrm{THz}$ pulses reflected by the sample and air (bare imaging window) respectively, $r_{\text {quartz-air }}$ is the reflection coefficient at the air-window interface, which is calculated by the Fresnel coefficients [30]. On the right-hand side, $Z_{\text {skin }}$ is the impedance of the skin which is determined by the water distribution profile [24] $W(z, t)$ as calculated by the flow chart shown in Figure S1. $\theta_{\text {quartz }}=13.64^{\circ}$ is the incident angle in the quartz window, which is calculated by Snell's law. $\varepsilon_{\text {quartz }}=(2.12-0 i)^{2}$ is the permittivity of quartz window in the $\mathrm{THz}$ frequency range.

\section{4 | Water diffusivity fitting}


The first measurement (immediately after the skin contacts the imaging window) is used to estimate the five initial parameters of the steady state skin: the thickness of stratum corneum and epidermis, the water concentration at the three interfaces: the skin surface, the interface between the SC and epidermis and the interface between the epidermis and dermis. The details are given in the supplementary information. By substituting the function of $D$ into the SC swelling model and stratified media theory, the $\mathrm{THz}$ reflectivity for occluded skin at various occlusion times is calculated. By fitting the modeled reflectivity to the measured reflectivity during the 20 minutes of occlusion and minimizing the objective function shown below, the parameters of $D$ in Eqn (5) are determined:

$$
o b j_{-} \text {fun }=\sum_{t=0}^{t=1200 s}\left\{\sum_{\text {freq }=0.3}^{\text {freq }=0.8} \mid R_{\text {cal }}(t, \text { freq })-R_{\text {meas }}(t, \text { freq }) \mid\right\}
$$

is the occlusion time, freq is the frequency, $R_{\text {cal }}$ and $R_{\text {meas }}$ are the modeled and measured reflectivity at occlusion time $t$, calculated by the right and left side of Eqn (6), respectively. We study the frequency range $0.3 \mathrm{THz}$ to $0.8 \mathrm{THz}$, as the Signal to Noise Ratio (SNR) of our system is the highest in this range and there are minimal scattering effects induced by the rough skin surface [24]. The summarized flow chart of the total calculating process is shown in Figure 1(b).
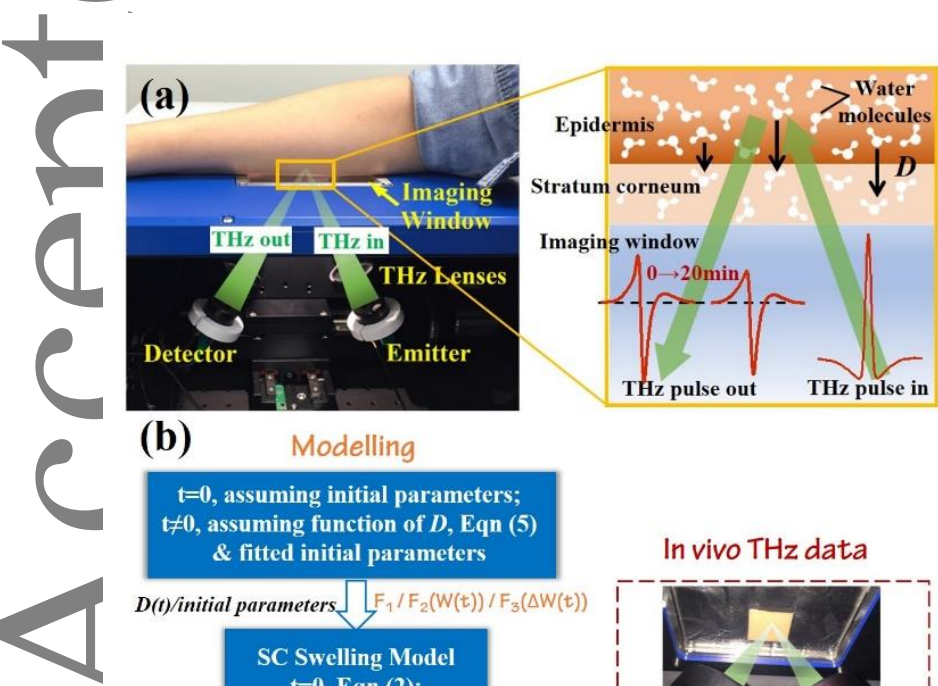

D(t)/initial parameters $\square F_{1} / F_{2}(W(t)) / F_{3}(\Delta W(t))$
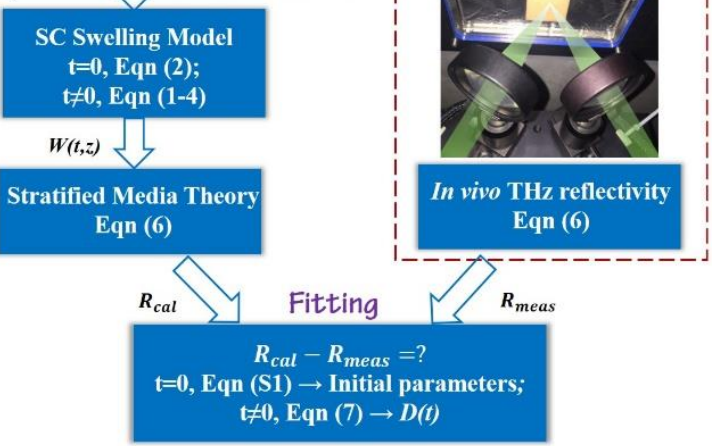

FIGURE 1 In vivo $\mathrm{THz}$ measurement and calculation process of the water diffusivity. (a) Schematic of the $\mathrm{THz}$ beam being incident on the layers of skin. (b) The flow chart of the calculation process of the water diffusivity $D$, the insert is the visible figure taken from beneath of the imaging window, the hole in the aluminium foil is used to help image registration.

\section{3 | RESULTS AND DISCUSSION}

\section{1 | THz response of occluded skin}

Figure 2(a) gives the $\mathrm{THz}$ response in the time domain at different occlusion times. The measured $\mathrm{THz}$ signals are processed to remove the signals with low SNR frequencies [23]. The pulses shown in Figure 2(a) are translated horizontally for display clarity. As occlusion time increases, the amplitude of $\mathrm{THz}$ signal decreases significantly; the first 100 seconds shows the largest decay. Figure 2(b) shows how the peak to peak of the pulses decreases over the 20 minutes of occlusion. A decrease is also observed in the $\mathrm{THz}$ reflectivity upto about $0.5 \mathrm{THz}$ (depending on occlusion duration) as shown in Figure 2(c-d). Above $0.5 \mathrm{THz}$, the reflectivity increases with frequency as the refractive index of the skin decreases with increasing frequency: it starts from higher than the refractive index of quartz $n_{\text {quartz }}(2.12)$, then decreases to close to $n_{\text {quartz }}$, then gradually drifts away from $n_{\text {quartz. }}$. From the Fresnel equation, we know that smallest reflected signal occurs from the quartz-skin system when $n_{\text {quartz }}=n_{\text {skin }}$. As the frequency increases beyond this, the difference between the refractive indices increases again, and so the amplitude of the reflected signal also increases. This trend is less pronounced for long occlusion times as the refractive index is flatter for more hydrated skin. In our previous work [23], we have shown that the temperature change of the skin and the imaging window has negligible effect on the $\mathrm{THz}$ signal, and that the effects of the perspiration layer on the imaging window can also be ignored. Consequently, we deduce that the substantial reduction in $\mathrm{THz}$ signal is caused by the increased water content in the skin increasing the absorption of THz light. Thus THz-TDRS is able to perform real-time monitoring of the water profile change in vivo and capture hydration fluctuations in the skin. 

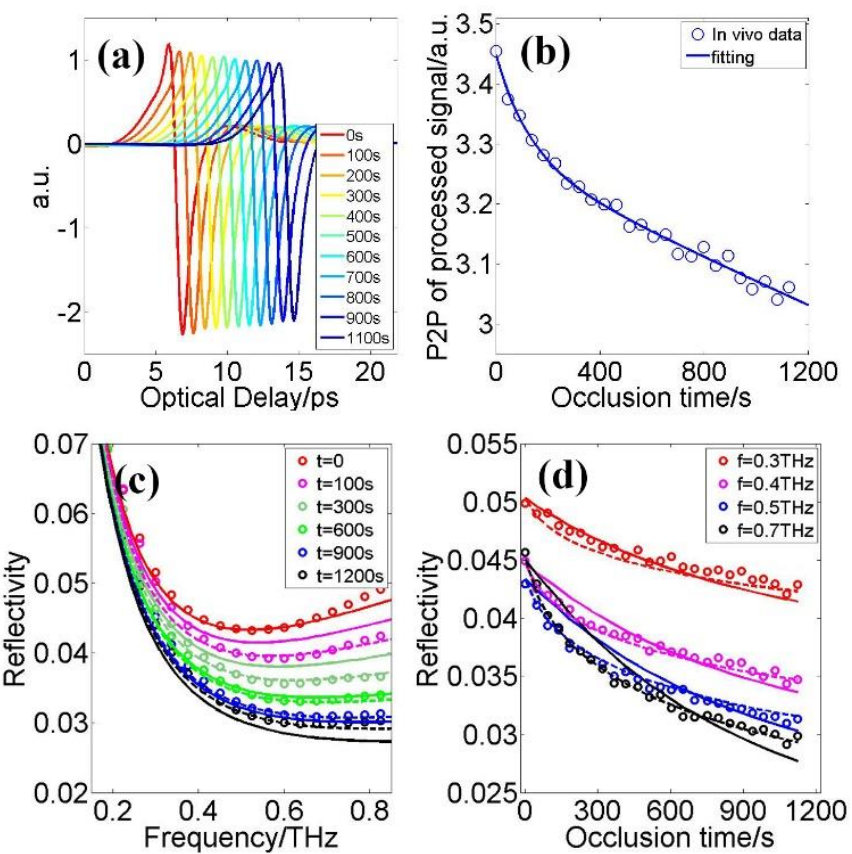

FIGURE $2 \mathrm{THz}$ time and frequency domain results during skin occlusion. (a) Processed THz signal with different occlusion times. (b) Peak to peak of the THz signal during 1200 seconds of occlusion. (c) The spectrum of calculated and measured reflectivity at different occlusion times. (d) The calculated and measured reflectivity at different frequencies as a function of occlusion time. Dots are in vivo measured data, solid and dashed lines are data calculated assuming either a constant $\left(F_{1}\right)$ or a water concentration correlated $\left(F_{3}\right)$ diffusivity, respectively. All the data is from subject 1 .

\subsection{Initial skin parameters}

TABLE 1 Fitted initial skin parameters of five subjects based on Eqn (S1).
For all five subjects, the definition of $F_{2}$ in Eqn (5) returns the results with $\alpha_{1}$ being 0 . We therefore deduce that the diffusivity of water in the skin does not correlate with the surface hydration level positively under occlusion. Table 2 details the calculated water diffusivity for definitions $F_{1}$ and $F_{3}$ for the 5 subjects as well as the values of the objective function shown in Eqn (7).

Figures 2(c-d) present the calculated $\mathrm{THz}$ reflectivity of the two functions of $\mathrm{D}$ for subject 1 . From the results shown in Table 2 and Figure 2(c-d), for all the five subjects, it is clear that the water concentration correlated diffusivity $F_{3}$ describes the water diffusion in the occluded skin more precisely than the constant diffusivity $F_{1}$, particularly at the onset of the occlusion. The averaged water concentration correlated diffusivity $\left(F_{3}\right)$ results of each subject are plotted in Figure 3 as a function of water concentration difference between the two surfaces of the SC.

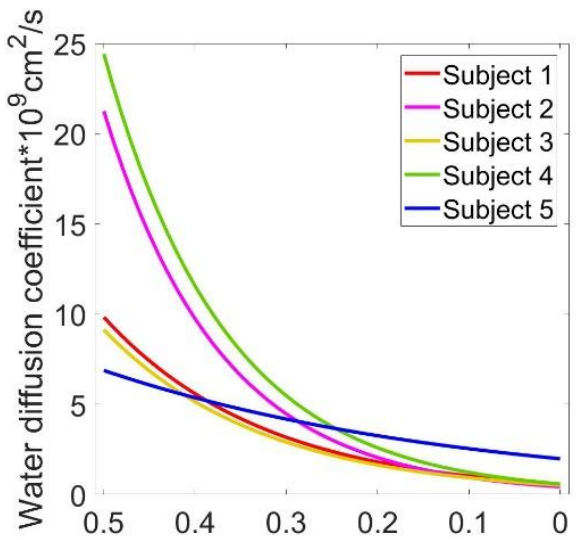

Water concentration difference $/ \mathrm{g} / \mathrm{cm}^{3}$

FIGURE 3 The calculated water diffusivity for five subjects as a function of the water concentration difference between the two

\begin{tabular}{|llllll} 
Subject & $\begin{array}{l}\text { SC thickness } \\
\left(\boldsymbol{L}_{\mathbf{0}}, \boldsymbol{\mu m}\right)\end{array}$ & $\begin{array}{l}\text { Epidermis } \\
\text { thickness }(\boldsymbol{\mu m})\end{array}$ & $\begin{array}{l}\text { Initial water } \\
\text { concentration at } \\
\text { the surface of SC } \\
\left(\boldsymbol{W}_{\boldsymbol{S 0} \mathbf{0}} \boldsymbol{g} / \mathbf{c m}^{\mathbf{3}}\right)\end{array}$ & $\begin{array}{l}\text { Initial water } \\
\text { concentration at } \\
\text { the bottom of SC } \\
\left(\boldsymbol{W}_{\boldsymbol{B} \mathbf{0}}, \boldsymbol{g} / \mathbf{c m}^{\mathbf{3}}\right)\end{array}$ & $\begin{array}{l}\text { Water concentration at the } \\
\text { bottom of epidermis }\left(\boldsymbol{g} / \mathbf{c m}^{\mathbf{3}}\right)\end{array}$ \\
\hline 1 & $23.24 \pm 1.01^{*}$ & $59.80 \pm 7.76$ & $0.16 \pm 0.01$ & $0.70 \pm 0.02$ & $0.74 \pm 0.01$ \\
\hline 3 & $25.84 \pm 1.34$ & $58.91 \pm 10.89$ & $0.16 \pm 0.01$ & $0.72 \pm 0.03$ & $0.77 \pm 0.01$ \\
\hline & $17.53 \pm 1.88$ & $66.34 \pm 13.84$ & $0.19 \pm 0.03$ & $0.74 \pm 0.01$ & $0.80 \pm 0.01$ \\
\hline & $22.39 \pm 0.58$ & $65.71 \pm 13.63$ & $0.19 \pm 0.02$ & $0.71 \pm 0.01$ & $0.75 \pm 0.01$ \\
\hline
\end{tabular}

$*$ mean $+/$-SD

The initial fitted skin parameter sets for the non-occluded state of five subjects are given in Table 1 . The variation of the fitted epidermis thickness is much larger than that of the other parameters, this is because this variable has little effect on the calculated $\mathrm{THz}$ reflectivity and so does not influence the results significantly.

\section{3 | Water diffusivity upon occlusion}

interfaces of the SC.

\subsection{Discussion on the calculated values of $D$}

\subsubsection{Comparison with the diffusivity in non-occluded skin}

The published diffusivity of water in non-occluded human skin determined by in vivo measurements or models has a wide range of values due to the different methods and instruments, namely $0.2-7.7 \times 10^{-9} \mathrm{~cm}^{2} / \mathrm{s}$ 
$[9,10,12,19,20,29]$. In this work, as shown in Table 2, for the constant assumption, the values of $D$ of the five subjects are all located in this range. For the water concentration correlated assumption, the diffusivity is larger than the upper boundary of the non-occluded range at the onset of the occlusion, then it gradually relaxes to the normal value range as the hydration level of the skin surface saturates to the hydration level at the deep skin.

Skin occlusion is known to affect the percutaneous transport of compounds and syntheses of molecules [3,7]. Therefore it may also alter the barrier function and physicochemical properties of the SC, which consequently causes a sudden reduction on the water resistance. This explains why the calculated water diffusivity in the occluded skin is higher than that in the non-occluded skin

\subsubsection{Comparison among three assumptions of $D$}

Different from the steady state skin [29,31], when skin is occluded, due to the diffusional water flux being blocked, the water discharge is no longer positively correlated with skin surface hydration. The fitting results of assumption $\boldsymbol{F}_{\mathbf{2}}$ support this argument. The better goodness of fit for function $\boldsymbol{F}_{\mathbf{3}}$ compared to $\boldsymbol{F}_{\mathbf{1}}$ confirms that the discharge of water decreases with decreasing water concentration gradient in the SC. This therefore shows that it is the water concentration gradient in the SC that drives the water diffusion from the inside to the outside. This observed exponential trend in the water diffusivity resembles other natural relaxation processes such as blood pressure and sweat rate recovery after physical exercise [32].

3.5 Convection velocity, water concentration and SC thickness change upon occlusion decreases. For the water concentration correlated case, the thickness of the SC increases to $31.8 \mu \mathrm{m}$ and the water concentration at the skin surface increases to $0.55 \mathrm{~g} / \mathrm{cm}^{3}$. The dynamic water concentration colormap under occlusion for subject 1 is given in Figure 4(c), corresponding to a water concentration correlated water diffusivity.
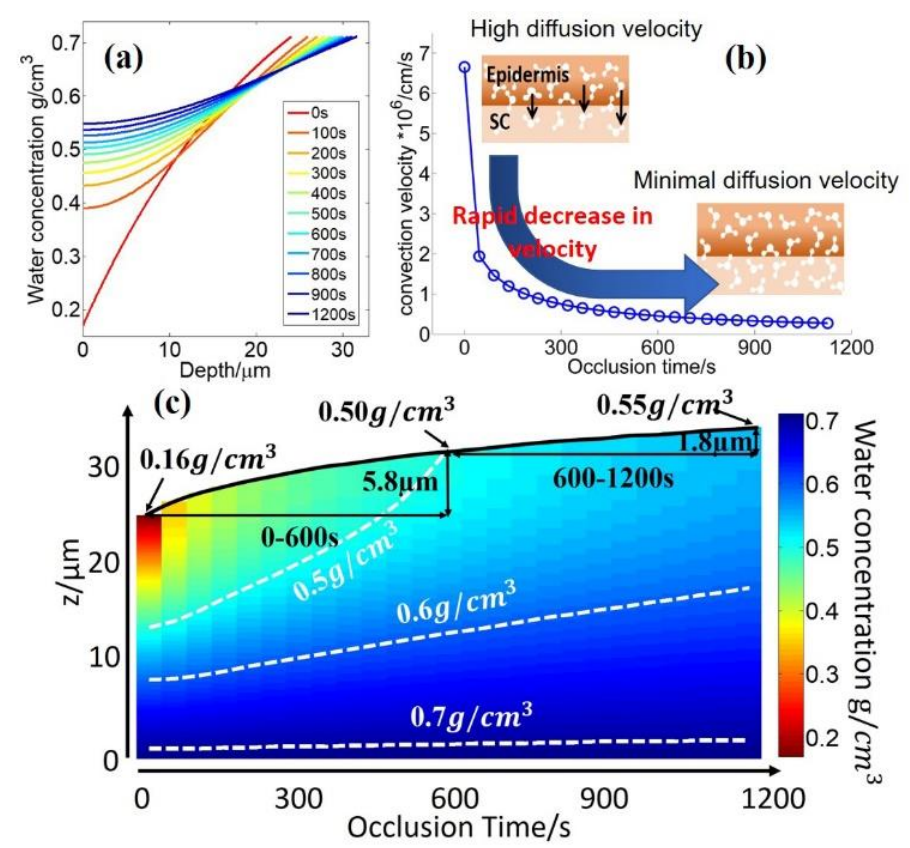

FIGURE 4 The calculated dynamic water concentration and velocity results upon occlusion. (a) The water concentration profile at different occlusion times. (b) The convection velocity as a function of the occlusion time. (c) The water concentration colormap

TABLE 2 Calculated parameters for the water diffusivity functions $F_{1}$ and $F_{3}$ of five subjects based on Eqn (7).

\begin{tabular}{|c|c|c|c|c|c|}
\hline \multirow[b]{2}{*}{ Subject } & \multicolumn{2}{|c|}{$F_{1}$} & \multicolumn{3}{|c|}{$F_{3}$} \\
\hline & $D_{0} \times 10^{9} \mathrm{~cm}^{2} / \mathrm{s}$ & $O b j_{f u n}$ in Eqn (7) & $D_{2} \times 10^{9} \mathrm{~cm}^{2} / \mathrm{s}$ & $\alpha_{2} \mathrm{~cm}^{3} / \mathrm{g}$ & $O b j_{f u n}$ in Eqn (7) \\
\hline & $2.38 \pm 0.08 *$ & $1.56 \pm 0.03$ & $0.58 \pm 0.13$ & $5.66 \pm 0.60$ & $0.70 \pm 0.08$ \\
\hline 2 & $3.08 \pm 0.10$ & $2.64 \pm 0.09$ & $0.43 \pm 0.09$ & $7.80 \pm 0.36$ & $0.75 \pm 0.06$ \\
\hline 3 & $1.97 \pm 0.16$ & $1.18 \pm 0.07$ & $0.52 \pm 0.07$ & $5.73 \pm 0.42$ & $0.71 \pm 0.02$ \\
\hline 4 & $2.99 \pm 0.17$ & $1.53 \pm 0.13$ & $0.58 \pm 0.09$ & $7.48 \pm 0.61$ & $0.82 \pm 0.08$ \\
\hline 5 & $3.49 \pm 0.15$ & $0.95 \pm 0.11$ & $1.98 \pm 0.23$ & $2.49 \pm 0.44$ & $0.63 \pm 0.16$ \\
\hline
\end{tabular}

* mean+/-SD

Figures $4(a-b)$ show the convection velocity (which is equal to the SC swelling velocity in this case) changing under occlusion and the results calculated from one initial parameter set of subject 1 on the water concentration profile change in the occluded SC. The water concentration at the skin's surface and the convection velocity change most dramatically at the onset of occlusion. As the occlusion time increases, the skin's surface becomes hydrated, the difference between the curves decreases and the water concentration gradient as a function of depth in skin (z) and occlusion time. The black curve shows how the thickness of the SC changes with occlusion time. The white dashed curves highlight the contour lines of the water concentration being $0.5,0.6$ and $0.7 \mathrm{~g} / \mathrm{cm}^{3}$. The data used are all from subject 1 , and assuming water diffusivity is positively correlated to the water concentration difference $\left(F_{3}\right)$.

\subsection{Error analysis}

3.6.1 | Robustness of in vivo THz-TDRS measurements 
The structure and morphology in skin varies with position [33] additionally, Rogiers et al found that a subject's dominant forearm tends to have higher TEWL than the other forearm and that skin tends to have higher TEWL in the evening than in the morning. [34]. Therefore, to make meaningful comparisons, as far as possible, we tried to measure exactly the same area of skin at the same time of day. Additionally, Chan et al have proved that compression affects the optical properties of human skin at wavelengths ranging from 400 to $1800 \mathrm{~nm}$ [35], we therefore tried to keep the pressure between the skin and the imaging window exactly the same for all the subjects in our measurements. Furthermore, some algorithms and designs for sample holders have been proposed to get more reliable and repeatable in vivo THz-TDRS results, and problems such as pressure being applied to the soft tissue variation still need to be fully resolved.

3.6.2 Initial parameters and water distribution

The initial water distribution in the SC is commonly assumed to linearly increase with the depth in skin models $[4,24]$, or assumed to be an approximately linear distribution, like Eqn (2) in this model. However, in practice the upper 34 - $\mathrm{m}$ near the skin's surface show different water distributions: the water concentration increases with a smaller gradient in this depth range, or it keeps nearly constant, or even shows a small dip at around $2 \mu \mathrm{m}[13,14,27,36]$. This slight difference might affect the fitting results of the initial water concentration on the skin surface: for example Figures 1 and 2 given in reference [12] give the surface water concentration measured by Raman spectroscopy (around $0.3 \mathrm{~g} / \mathrm{cm}^{3}$ ) to be higher than that in the approximately linear fitted model (around $0.2 \mathrm{~g} / \mathrm{cm}^{3}$ ). The difference in the skin's upper $3-4 \mu \mathrm{m}$ layers explains why the fitted initial surface water concentration in Table 1 is a bit lower than the literature values. Although the initial parameters of the nonoecluded skin can be determined by the data collected at occlusion time being zero and fitting it to the SC swelling and stratified media models, it would be better to measure them by other more direct techniques.

\subsection{Potential applications}

The hydration effects caused by occlusion disturb percutaneous transport [7], this work therefore has the potential to quantitatively measure compound diffusion and skin absorption processes which could be useful for developing transdermal treatments. Additionally, healthy or barrier function damaged skin may have different reactions to occlusion which may aid skin diagnosis.

As an example, we show in Figure 5 how the $\mathrm{THz}$ response of skin significantly changes after applying a silicone plaster (designed to improve scar healing) for 3 hours The peak to peak signal and the $\mathrm{THz}$ reflectivity under occlusion after the silicone plaster has been worn are much lower than before. The data suggest that the water content in the skin increases when the plaster is worn, and that this also affects the occlusion response. In this way, it would therefore be possible to quantitatively evaluate and develop skin treatments.
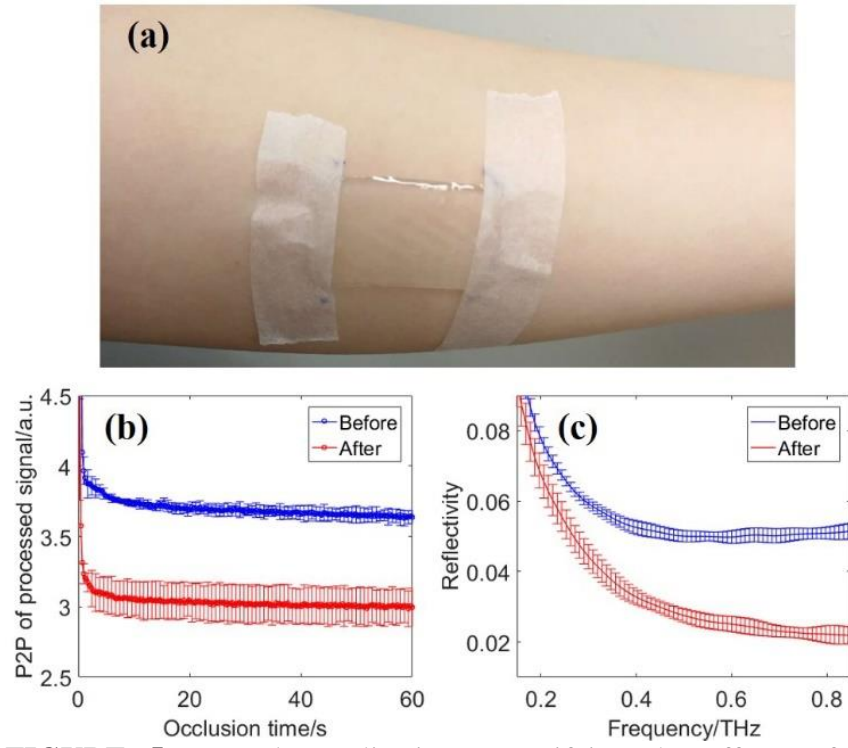

FIGURE 5 Example application: quantifying the effects of a silicone plaster. (a) Photograph of the silicone plaster applied to normal skin. (b) Peak to peak of the processed signal during 60 seconds of occlusion for normal skin before (blue) and after (red) applying a silicone plaster. (c) Reflectivity of normal skin before (blue) and after (red) applying a silicone plaster. Three subjects were each measured 3 times both before and after, the mean is plotted and the error bars are the standard deviation.

\section{4 | CONCLUSION}

In this work, we show that in vivo THz-TDRS is able to detect subtle hydration changes in human skin induced by occlusion. The accumulation of water in the SC reduces the reflected $\mathrm{THz}$ signal, and is significant within seconds of the onset of occlusion. To obtain the dynamic water concentration profile in the occluded SC, we proposed a finite difference method to solve the SC swelling model for the occlusion case. We found the best fit to the data was $F_{3}$ in which the water diffusivity is positively correlated with the water gradient in the SC. This suggests that the water gradient in the SC drives the water discharge in the SC.

To our knowledge, this is the first time that the water diffusivity in occluded skin has been estimated. Our THz data show that skin occlusion may alter the barrier function and water resistance of the SC immediately. This is helpful for understanding how skin occlusion quantitatively affects scar healing, chemical absorption and molecular syntheses. For completeness, we conducted our study over a period of 20 minutes of occlusion, but there were significant changes even within the first 30 seconds of occlusion. Thus $\mathrm{THz}$ in vivo imaging has the potential to be a simple, quick and quantitative measure of skin diffusivity. In the future, more studies about percutaneous transport are expected to be investigated based on this work.

\section{ACKNOWLEDGEMENTS}

The authors would like to thank the Research Grants Council of Hong Kong (project numbers 14206717, 14205514 and 14201415), The Hong Kong Innovation and Technology Fund (project number ITS/371/16), The Royal Society 
Wolfson Merit Award and the Hong Kong PhD Fellowship Award for partial support of this work.

\section{REFERENCES}

[1] I.H. Blank, J. Moloney, A.G. Emslie, I. Simon, C. Apt, Journal of Investigative Dermatology 82 (1984) 188.

[2] J. Faergemann, R. Aly, D. Wilson, H. Maibach, Archives of dermatological research 275 (1983) 383.

H. Zhai, H.I. Maibach, Skin Research and Technology 8 (2002) 1.

W. Pan, X. Zhang, M. Lane, P. Xiao, International journal of cosmetic science 37 (2015) 395.

[5] B. Berman, O.A. Perez, S. Konda, B.E. Kohut, M.H. Viera, S. Delgado, D. Zell, Q. Li, Dermatologic surgery 33 (2007) 1291. Y. Sawada, K. Sone, British journal of plastic surgery 45 (1992) 599.

H. Zhai, H.I. Maibach, Skin Pharmacology and Physiology 14 (2001) 1.

R.J. Scheuplein, I.H. Blank, Physiol Rev 51 (1971) 702.

Y.N. Kalia, F. Pirot, R.H. Guy, Biophysical journal 71 (1996) 2692.

[10] D.A. Schwindt, K.P. Wilhelm, H.I. Maibach, Journal of investigative dermatology 111 (1998) 385.

X. Li, R. Johnson, B. Weinstein, E. Wilder, E. Smith, G.B. Kasting, Chemical Engineering Science 138 (2015) 164.

[12] S. Bielfeldt, V. Schoder, U. Ely, A. van der Pol, J. de Sterke, K.-P. Wilhelm, International Journal of Cosmetic Science 31 (2009) 479.

M. Egawa, T. Hirao, M. Takahashi, Acta dermato-venereologica 87 (2007) 4.

[14] P. Caspers, G. Lucassen, H. Bruining, G. Puppels, Journal of Raman spectroscopy 31 (2000) 813.

[15] J.W. Fluhr, S. Sassning, O. Lademann, M.E. Darvin, S. Schanzer, A. Kramer, H. Richter, W. Sterry, J. Lademann, Experimental dermatology 21 (2012) 130.

[16] A.K. Dabrowska, C. Adlhart, F. Spano, G.M. Rotaru, S. Derler, L. Zhai, N.D. Spencer, R.M. Rossi, Biointerphases 11 (2016) 031015.
[17] J.A. Bouwstra, A. de Graaff, G.S. Gooris, J. Nijsse, J.W. Wiechers, A.C. van Aelst, Journal of Investigative Dermatology 120 (2003) 750.

[24] D.B. Bennett, W. Li, Z.D. Taylor, W.S. Grundfest, E.R. Brown, IEEE Sensors Journal 11 (2011) 1253.

[25] M. Saviz, O. Spathmann, J. Streckert, V. Hansen, M. Clemens, R. Faraji-Dana, IEEE Transactions on Terahertz Science and Technology 3 (2013) 635.

[26] E. Pickwell, B.E. Cole, A.J. Fitzgerald, M. Pepper, V.P. Wallace, Physics in medicine and biology 49 (2004) 1595.

[27] J. Nikolovski, G.N. Stamatas, N. Kollias, B.C. Wiegand, The Journal of investigative dermatology 128 (2008) 1728.

[28] G.D. Smith, Numerical solution of partial differential equations: finite difference methods, Oxford university press, 1985.

[29] X. Li, R. Johnson, G.B. Kasting, Journal of Pharmaceutical Sciences 105 (2016) 1141.

[30] X. Chen, E.P. Parrott, B.S.-Y. Ung, E. PickwellMacPherson, IEEE Transactions on Terahertz Science and Technology (2017).

[31] E.R. Cooper, B.F. Van Duzee, J Soc Cosmet Chem 76 (1976) 555.

[32] Y. Feldman, A. Puzenko, P.B. Ishai, A. Caduff, I. Davidovich, F. Sakran, A.J. Agranat, Physics in medicine and biology 54 (2009) 3341.

[33] X. Liang, S.A. Boppart, IEEE Transactions on Biomedical Engineering 57 (2010) 953.

[34] V. Rogiers, Skin Pharmacology and Physiology 14 (2001) 117.

This article is protected by copyright. All rights reserved. 
[35] E.K. Chan, B. Sorg, D. Protsenko, M. O'Neil, M. Motamedi, A.J. Welch, IEEE Journal of selected topics in quantum electronics 2 (1996) 943.

[36] R.R. Warner, M.C. Myers, D.A. Taylor, Journal of Investigative Dermatology 90 (1988) 218.

\section{CaUthor BiographIES}

- Please see Supporting Information online.

$1)$

SUPPORTING INFORMATION

Please see Supporting Information online.

rets

\section{(1)}

Graphical Abstract for Table of Contents

By exploiting in vivo terahertz reflection spectroscopy and skin models, this work develops a method to determine water diffusivity in occluded human skin for the first time. Dynamic water concentration and stratum corneum thickness changes upon occlusion are also tracked quantitatively. This work helps study the barrier function of the stratum corneum and will provide a new quantitative measure for use in skin studies.
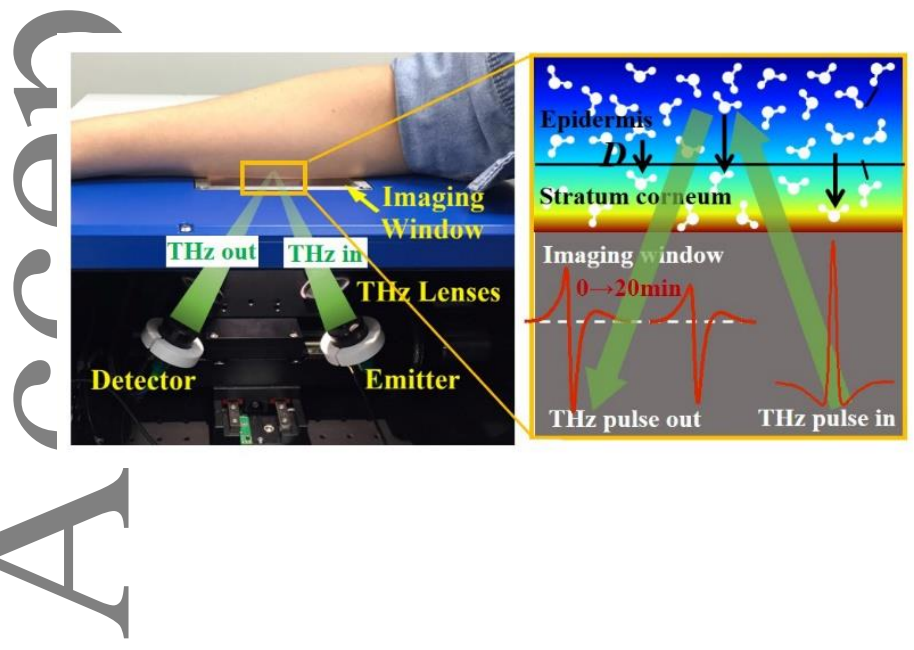

This article is protected by copyright. All rights reserved. 